\title{
Controlling the elastic modulus of cellulose nanofibril hydrogels—scaffolds with potential in tissue engineering
}

\author{
Kristin Syverud • Sigurd R. Pettersen • \\ Kurt Draget • Gary Chinga-Carrasco
}

Received: 7 August 2014/Accepted: 6 October 2014/Published online: 17 October 2014

(C) The Author(s) 2014. This article is published with open access at Springerlink.com

\begin{abstract}
Cellulose nanofibrils (CNF) form hydrogels at low concentrations. These hydrogels are held together by transient interactions such as entanglement of fibrils, non-specific ionic interactions and hydrogen bonds; and are thus vulnerable for changes in the chemical environment or the influence of mechanical forces. By a covalent crosslinking of the fibrils, stable permanent gels can be formed. In this study we have produced CNF by using TEMPO mediated oxidation followed by fibrillation. During this procedure, carboxyl and aldehyde groups are introduced on the CNF surfaces. The aldehyde groups are suitable sites for crosslinking, as aldehydes readily form covalent bonds to primary amines through formation of Schiff bases. For this purpose the diamines ethylenediamine and hexamethylenediamine, differing with four carbon atoms in the chain, were used as crosslinker molecules. The results show that by varying the concentration and length of the crosslinker molecules, the elastic modulus of the gels
\end{abstract}

K. Syverud $(\bowtie) \cdot$ G. Chinga-Carrasco

Paper and Fibre Research Institute, Høgskoleringen 6B,

7491 Trondheim, Norway

e-mail: kristin.syverud@pfi.no

K. Syverud · S. R. Pettersen

Department of Chemical Engineering, NTNU,

7491 Trondheim, Norway

K. Draget

Department of Biotechnology, NTNU, 7491 Trondheim, Norway could be controlled. The reversible gels were in this way transformed to irreversible gels by a simple water based reaction. Controlling gel strength is one important premise for the use of CNF in applications such as tissue engineering.

Keywords Cellulose nanofibrils · Nanocellulose · Hydrogels · Tissue engineering · Crosslinking

\section{Introduction}

Hydrogels are three-dimensional (3D) networks of mainly hydrophilic polymers capable of absorbing up to thousands times their own dry weight in water (Hoffman 2001; Seliktar 2012). In medicine, hydrogel products based on synthetic and natural polymers have had a large impact on patient care in recent years. Examples of products are soft contact lenses, biological adhesives used in surgical procedures and wound dressings. As hydrogels improve through better design, their use is likely to expand in applications such as drug-delivery devices, biosensors and as scaffolds for tissue engineering. In addition to the high water content of hydrogels, there is a range of other properties that are important for successful development of such applications, e.g. degradability in the human body, bioadhesion, bioactivity, transport through the network, and mechanical properties that should match those of native tissue (Seliktar 2012). 
In tissue engineering living cells are used to restore damaged tissue, and for this purpose scaffolds with tailored properties must be developed. Tissue engineering is much about imitating the extracellular matrix (ECM), which provides structural and biochemical support to the surrounding cells. One important factor of tissue scaffolds is the mechanical properties. The optimal mechanical properties for a cell niche vary as much as 300 -fold from soft brain tissue (elastic modulus, $\mathrm{E}=0.1 \mathrm{kPa}$ ) to rigid calcifying bone $(\mathrm{E}>30 \mathrm{kPa})$. There is a dynamic, complex interplay between the $3 \mathrm{D}$ environment of the ECM and the living cells. Cells attach to the ECM through a vast array of adhesive proteins, pull against the matrix and feel the resistance to deformation by the adjacent environment. Mechanically sensitive proteins along this connection will provide feedback, actuating processes in the cell (Discher et al. 2005; Reilly and Engler 2010). In a study of Engler et al. (2006), it was demonstrated how the elasticity of the matrix influences the development of stem cells into differentiated cell types. Polyacrylamid gels varying in elasticity were used as matrix for naive mesenchymal stem cells. It was proven that the matrix elasticity specified lineage towards neurons, myoblasts and osteoblasts. These results demonstrated how important it is to control the elasticity of the matrix in tissue engineering.

Cellulose nanofibrils (CNF) are nanoscaled fibres or fibrils of cellulose, normally produced from wood or annual plants. As dispersions of CNF form hydrogels at low concentrations, one suggested application area is as 3D tissue scaffolds (Bhattacharya et al. 2012; Lou et al. 2014). Several procedures for preparation of CNF from cellulose pulp exist, among them TEMPOmediated oxidation using sodium hypochlorite as oxidant (Saito et al. 2006). In this method, aldehyde and carboxyl groups are introduced on the surfaces of the cellulose fibrils. TEMPO mediated oxidation facilitates the separation of nanofibrils by mechanical shear forces. This results in a dispersion of individualized nanofibrils, with widths of typically $3.5-20 \mathrm{~nm}$ (Chinga-Carrasco et al. 2011), and length in the $\mu \mathrm{m}$ range (e.g. estimated to be between 0.2 and $2.2 \mu \mathrm{m}$ depending on oxidation and homogenization conditions; Ishii et al. 2011; Fukuzumi et al. 2013). At low solid content (approx. $0.5 \%$ ), the fibril dispersions are reversible or physical gels, held together by fibril entanglement, ionic interactions and hydrogen bonds.
Reversible gels, also known as weak or false gels, are usually not robust, and can easily be destroyed by changes in the chemical environment or if they are subjected to mechanical forces. They can even be diluted away by adding more solvent. Permanent or chemical gels can be formed by covalently crosslinking the fibrils. Nge et al. (2010) reported the crosslinking of bacterial cellulose (BC) with chitosan through carbodiimide-mediated amide binding. $\mathrm{BC}$ has a nonuniform pore structure and too small pores for use as tissue scaffolds (Nge et al. 2010). Thus, they disintegrated the BC and introduced surface carboxyl groups through TEMPO mediated oxidation to develop structures suitable for this application.

A previous study showed that aldehyde groups on oxidized CNF, can be used to crosslink the fibrils and obtain permanent gel-structures (Syverud et al. 2011). This was done by linking aldehyde groups on the fibrils by adding either polyethyleneimine (PEI) or poly $\mathrm{N}$-isopropylacrylamide-co-allylamine-co-methylenebisacrylamide (pNIPA) and then cryo-gelling the samples. In another study small crosslinker molecules were used to crosslink cellulose fibres (Han et al. 2010). Aqueous triamino-1,3,5-triazine (melamine) solutions were used to crosslink the fibres in lyocell fabrics through the creation of Schiff-bases. A Schiff base is the double bond which is created between the nitrogen on a primary amine containing molecule and the carbon on an aldehyde containing molecule. The double bond can be stabilized by reduction to a secondary amine linkage. This reduction can be done by a reduction agent such as sodium borohydride $\left(\mathrm{NaBH}_{4}\right.$; Han et al. 2010).

In the present work we focus on crosslinking of $\mathrm{CNF}$ and controlling the elastic modulus of CNF gels. Only water based systems are used. The experimental design include two steps; (1) production of CNF using TEMPO mediated oxidation, and (2) crosslinking the $\mathrm{CNF}$ by amine-aldehyde reactions and ionic interactions. The aldehyde groups introduced through the production process of CNF are utilized in the crosslinking with diamines. Diamines are commonly used as crosslinking agents in biomedical research (Barbucci et al. 2000), and we have chosen ethylenediamine (EDA) and hexamethylenediamine (HMDA), differing only by the length of the carbon chain and hence also the spacing distance between the functional amino groups. By altering the crosslinker molecules and varying the concentration, we demonstrate how 
the elastic modulus of CNF based hydrogels can be controlled.

\section{Materials and methods}

Chemicals

All chemicals used in this study were of laboratorygrade quality purchased from Sigma Aldrich. The water used was of Milli-Q quality (Elga USF, Maxima, resistivity $18.2 \mathrm{M} \Omega \mathrm{cm}$ ).

\section{Production of cellulose nanofibrils}

Cellulose pulp from softwood was used as raw material for production of fibrils. CNF were produced using 2,2,6,6-tetramethylpiperidinyl-1-oxyl (TEMPO) and sodium bromide $(\mathrm{NaBr})$ to catalyze the oxidation of primary alcohol groups with $\mathrm{NaClO}\left(3.8 \mathrm{mmol} \mathrm{g}^{-1}\right.$ cellulose; Saito et al. 2006). The $\mathrm{pH}$ was kept constant at 10.5 by adding sodium hydroxide $(\mathrm{NaOH})$ during the reaction. When the $\mathrm{pH}$ stopped decreasing, the reaction was stopped and the $\mathrm{pH}$ adjusted to seven by addition of hydrochloric acid ( $\mathrm{HCl})$. The pulp was washed by filtration using dionized water. The oxidized kraft pulp ( $1 \%$ consistency) was homogenized with a Rannie 15 type 12.56X homogenizer (APV, SPX Flow Technology, Silkeborg, Denmark), operated at 1,000 bar pressure. The fibrillated material was collected after one pass through the homogenizer. The yield of the fibrillated material was estimated using a FiberMaster device (Chinga-Carrasco et al. 2014). Toxicity of CNF produced by using this procedure has been studied, and no toxic effects have been observed (Alexandrescu et al. 2013).

Determination of carboxylic acids and aldehyde groups

The content of carboxyl and aldehyde groups was determined by conductometric titration, in a procedure similar to a previously described method (Saito and Isogai 2006). $16.6 \mathrm{~mL} 0.01 \mathrm{M} \mathrm{NaCl}$ was added to a CNF dispersion containing $0.1 \mathrm{~g}$ solid content, which was further diluted with water to a total volume of $200 \mathrm{~mL}$. The $\mathrm{pH}$ was adjusted to approx. 2.8 by addition of $0.1 \mathrm{M} \mathrm{HCl}$. Titration was performed with $0.04 \mathrm{M}$ $\mathrm{NaOH}$ solution added at a rate of $0.1 \mathrm{~mL} \mathrm{~min}^{-1}$ up to
$\mathrm{pH}$ of approximately 11 using an automatic titrator (Metrohm 902 Titrando). The conductivity of the sample was automatically measured at increments of $0.01 \mathrm{~mL}$ using a Metrohm 856 Conductivity Module, and the data was recorded by Tiamo ${ }^{\circledR}$ Titration Software. The carboxylate content was calculated from the titration curve (Gran plot). This analysis was also done after oxidation of aldehyde groups to carboxyl groups with $\mathrm{NaClO}_{2}$ (Saito and Isogai 2006). To avoid the influence of acetic acid residues on the carboxylate level, the $\mathrm{NaClO}_{2}$ oxidized $\mathrm{CNF}$ dispersion was washed by centrifuging five times at $2,700 \mathrm{rpm}$, with the supernatant being replaced by deionized water between each centrifugation. With the assumption that all aldehyde groups are oxidized, the difference in carboxylate content before and after the $\mathrm{NaClO}_{2}$ oxidation yields the aldehyde content.

Preparation of hydrogels by crosslinking

The two diamines (EDA and HMDA), having amine functional groups in both ends and differing only by four carbons in the hydrocarbon chain, were chosen as crosslinker molecules. Amines react readily with aldehydes, and thus the diamines were expected to make covalent bonds to aldehydes formed on the CNF surfaces as a result of the TEMPO oxidation. EDA and HMDA were dissolved in ionized water to concentrations of $0.005,0.05$, and $0.15 \mathrm{~g} \mathrm{~mL}^{-1}$. The volumes of crosslinker solution that were needed to yield the concentrations shown in Table 1 (mmol crosslinker per g CNF) were found by stoichiometric calculations and added to the original CNF dispersions using micropipettes. Deionized water was added so that the final CNF content of all samples was $0.80 \%$. To some of the samples the reduction agent 2-picoline-borane was added. This reduction agent has been suggested as an alternative to sodium borohydride reduction $(\mathrm{Ru}-$ haak et al. 2010). 2-picoline-borane (2-Methylpyridine borane complex solution $95 \%$ ) was dissolved in $96 \%$ ethanol to a concentration of $0.042 \mathrm{~g} \mathrm{~mL}^{-1}$, as the reduction agent was found to react violently when dissolved directly in water. This solution was added to a separate concentration series with HMDA, to a concentration of $1.22 \mathrm{mmol}$ reduction agent per $\mathrm{g}$ $\mathrm{CNF}$.

The samples were stirred for approximately $5 \mathrm{~min}$ and casted into cylindrical, stainless steel molds of $18 \mathrm{~mm}$ height and with $16 \mathrm{~mm}$ in inner diameter. The 
Table 1 Overview of crosslinked hydrogel samples

\begin{tabular}{lll}
\hline Sample code & Crosslinker & $\begin{array}{l}\text { Crosslinker concentration } \\
\left(\mathrm{mmol} \mathrm{g}^{-1} \mathrm{CNF}\right)\end{array}$ \\
\hline CNF & - & 0 \\
E1 & EDA & 0.061 \\
E2.5 & EDA & 0.152 \\
E5 & EDA & 0.304 \\
E10 & EDA & 0.609 \\
E50 & EDA & 3.042 \\
E100 & EDA & 6.084 \\
H1 & HMDA & 0.061 \\
H2.5 & HMDA & 0.152 \\
H5 & HMDA & 0.304 \\
H10 & HMDA & 0.612 \\
H50 & HMDA & 3.046 \\
H100 & HMDA & 6.092 \\
\hline
\end{tabular}

The number in the sample code implies the ratio of crosslinker concentration of the sample compared to the lowest crosslinker concentration

molds were sealed and placed overnight in a heating cabinet at $80{ }^{\circ} \mathrm{C}$. The time for reaction varied from 17 to $20 \mathrm{~h}$.

Small deformation oscillation rheology

Rheological assessment was performed using a shear rheometer (Stresstech, Reologica Instruments $\mathrm{AB}$, Sweden). Cone-plate measurement geometry (cone $=$ $4^{\circ}$ gradient, diameter $=35 \mathrm{~mm}$, gap $=150 \mu \mathrm{m}$ at center) was used for all measurements. Amplitude sweeps were done in order to determine the range of the linear viscoelastic regime in three CNF dispersions of varying concentration $(0.98,0.80$ and $0.50 \%)$. The strain was increased logarithmically from 0.001 to 1 , while the frequency and temperature were kept constant at $1 \mathrm{~Hz}$ and $20^{\circ} \mathrm{C}$. For studying the crosslinking reactions, i.e. the gelling process, HMDA solution was added to a CNF dispersion to a concentration of $3.046 \mathrm{mmol} \mathrm{g}^{-1} \mathrm{CNF}$. This dispersion was further diluted to a CNF content of $0.80 \%$ and added directly to the rheometer. The samples were covered with silicone oil (Dow Corning ${ }^{\circledR}, 200 / 10$ cS fluid, UK) to prevent evaporation during the measurements. During the rheological assessment, the temperature was increased to $80{ }^{\circ} \mathrm{C}$ and kept constant for $60 \mathrm{~min}$ before being lowered to the initial temperature of $20^{\circ} \mathrm{C}$. The temperature gradient during the increase/decrease was $\pm 4^{\circ} \mathrm{C} \mathrm{min}^{-1}$. The strain and frequency was 0.01 and $1 \mathrm{~Hz}$, respectively. CNF samples without crosslinker were subjected to the same assessment in order to study the effect of the crosslinking.

Longitudinal compression measurements

To determine the Young's modulus (E) of the cylindrical cast gels, a texture analyzer (TA.XT.-Plus Texture Analyzer, Stable Micro Systems, Surrey, UK) was used to perform compression measurements. The compression speed was $0.1 \mathrm{~mm} \mathrm{~s}^{-1}$, and the compression force was measured by a $1 \mathrm{~kg}$ load cell. The gels were compressed $2 \mathrm{~mm}$ in longitudinal direction after having reached a trigger load of $1 \mathrm{~g}$. The compression force was plotted as a function of deformation length, and linear regression was performed on the data points from 0.15 to $0.30 \mathrm{~mm}$ compression to find the gradient in the linear region. Since Young's modulus is proportional to the initial gradient of the longitudinal stress-strain curve, it was calculated by multiplying the gradient with the initial height and dividing by the initial cross sectional area of the gels.

\section{AFM and SEM}

For investigating the dimensions of the CNFs, thin films were prepared by drying a $0.25 \% \mathrm{CNF}$ dispersion at room temperature. The surface topography of the films was imaged using an atomic force microscope (diMultiMode V AFM) with a NanoScope V controller (Veeco Instruments Inc., Santa Barbara, CA, USA). The imaged area was $2,000 \mathrm{~nm} \times 2,000 \mathrm{~nm}$, with an image size of $1,024 \times 1,024$ pixels, and all images were acquired using ScanAsyst mode in air at room temperature.

Pieces of freeze dried gels (H1, H50, E1, E50 and pure CNF) were imaged by a Hitachi S-3000 N scanning electron microscope (SEM). Pieces from the edges which had been facing the casting mold walls, as well as pieces with the cross sectional area facing upwards, were attached to appropriate sample holders by double sided conductive carbon tape. To improve the signal and to avoid charge accumulation, the samples were coated with gold for $45 \mathrm{~s}$ using an Agar Automatic Sputter Coater (Agar Scientific, UK). The samples were imaged with a secondary electron detector at an acceleration voltage of $5 \mathrm{kV}$. 


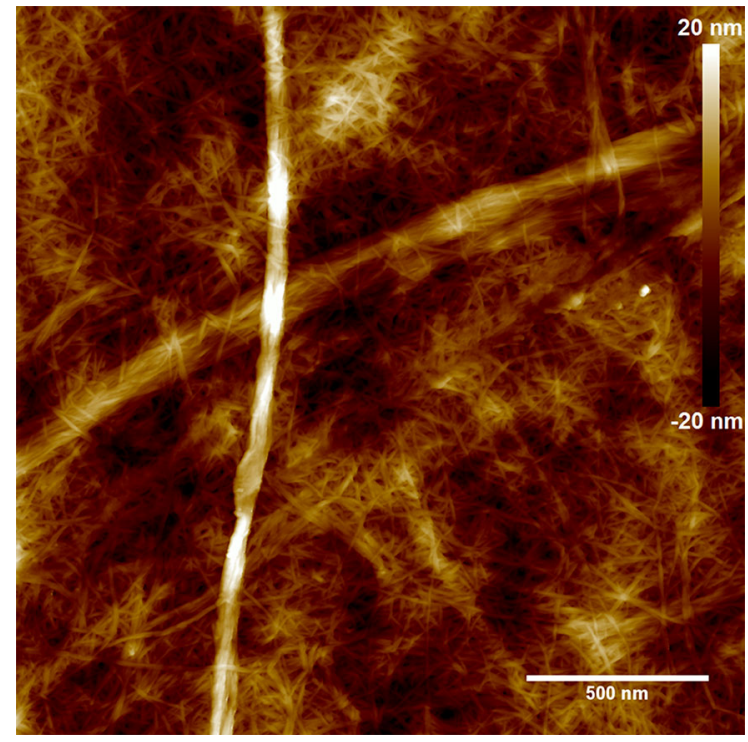

Fig. 1 AFM image of the CNF produced using TEMPO mediated oxidation followed by homogenization. The fibrillation degree was determined to be $83 \%$ according to ChingaCarrasco et al. 2014, and the fibril suspension was used without further fractionation

\section{Water reabsorption}

To investigate the stability of the crosslinked gels, the samples were immersed in water and soaked for approximately $5 \mathrm{~h}$. Some gels were also subjected to violent magnetic stirring. Freeze-dried gels (E50, H50 and pure $\mathrm{CNF}$ ) were immersed in MQ-water and taken out at different times to determine water absorption gravimetrically. Using tissue paper, secreted and surplus water was soaked up before weighing. The water absorption was estimated according to Eq. 1:

Water absorption $(\%)=\frac{(W s-W d)}{W d} \times 100$

where $W s$ and $W d$ correspond to the weight of the wetted and dry sample, respectively.

\section{Results and discussion}

CNF was successfully produced by TEMPO mediated oxidation followed by fibrillation as can be observed in Fig. 1. The yield of the produced CNF was estimated to be $83 \%$. The amount of carboxyl and aldehyde groups was determined to be $0.81 \pm 0.03 \mathrm{mmol} \mathrm{g}^{-1} \mathrm{CNF}$ and $0.18 \pm 0.04 \mathrm{mmol} \mathrm{g}^{-1} \mathrm{CNF}$, respectively. The
Table 2 Storage $\left(\mathrm{G}^{\prime}\right)$ and loss $\left(\mathrm{G}^{\prime \prime}\right)$ moduli for CNF dispersions at different concentrations

\begin{tabular}{lcc}
\hline CNF consistency $(w \%)$ & $\mathrm{G}^{\prime}(\mathrm{Pa})$ & $\mathrm{G}^{\prime \prime}(\mathrm{Pa})$ \\
\hline 0.50 & $31.8 \pm 1.0$ & $7.1 \pm 0.9$ \\
0.80 & $126 \pm 12$ & $16 \pm 2$ \\
0.98 & $275 \pm 62$ & $43 \pm 13$ \\
\hline
\end{tabular}

aldehyde groups were utilized in the subsequent covalent crosslinking. CNF dispersions are physical gels at low solid content. Table 2 shows the storage $\left(\mathrm{G}^{\prime}\right)$ and loss $\left(\mathrm{G}^{\prime \prime}\right)$ moduli for $\mathrm{CNF}$ dispersions with varying concentrations. Amplitude sweeps showed that for strains $<0.04$ a linear viscoelastic regime was found for all measured concentrations of CNF. The storage $\left(\mathrm{G}^{\prime}\right)$ and loss $\left(\mathrm{G}^{\prime \prime}\right)$ moduli of the CNF dispersions were averaged for strains from 0.001 to 0.04 for each concentration.

In order to study the crosslinking reaction, a blend of CNF and HMDA crosslinker molecules was subjected to rheological assessment immediately after mixing. During the assessment the temperature was increased to $80^{\circ} \mathrm{C}$ and kept at this level for $1 \mathrm{~h}$. A reference sample without crosslinker was assessed for comparison. The results are given in Fig. 2. The increase in storage modulus showed clearly that the crosslinking started to take place when the temperature was increased. The storage modulus increased steadily as a function of temperature, and by more than 11 times during the $80 \mathrm{~min}$ the experiment lasted. The reference sample without crosslinker had nearly constant storage and loss moduli as expected.

For determination of Young's modulus (E) of the gels, all samples in Table 1 were subjected to compression measurements using a texture analyzer. For the samples crosslinked with HMDA, a series having the reductive agent 2-picoline-borane was also added. 2-picoline-borane was chosen since it reduces the Schiff-bases to amines without reducing non-reacted aldehyde groups, thus making it suitable for one-pot reactions (Ruhaak et al. 2010). This is an advantage compared to the more commonly used sodium borohydride that also reacts with aldehyde groups, or the milder reduction agent sodium cyanoborohydride which do not reduce aldehyde groups, but is lethally toxic. The results (Fig. 3) show clearly that there is a relationship between crosslinker concentration and $\mathrm{E}$, and that $\mathrm{E}$ starts to level out after approximately 0.6 mol crosslinker $\mathrm{g}^{-1} \mathrm{CNF}$. In addition, the length 
Fig. 2 Temperature sweep, 20-80-20 ${ }^{\circ} \mathrm{C}$

$\left( \pm 4{ }^{\circ} \mathrm{C} \mathrm{min}{ }^{-1}\right)$, constant $80{ }^{\circ} \mathrm{C}$ for $1 \mathrm{~h}$, measured on $0.80 \% \mathrm{CNF}$ with and without HMDA (H) $\left(3.05 \mathrm{mmol} \mathrm{g}^{-1} \mathrm{CNF}\right)$. The frequency and strain was held constant at $1 \mathrm{~Hz}$ and 0.01 , respectively. At the last data points, the storage modulus of the crosslinked gel is approximately $1,120 \mathrm{~Pa}$
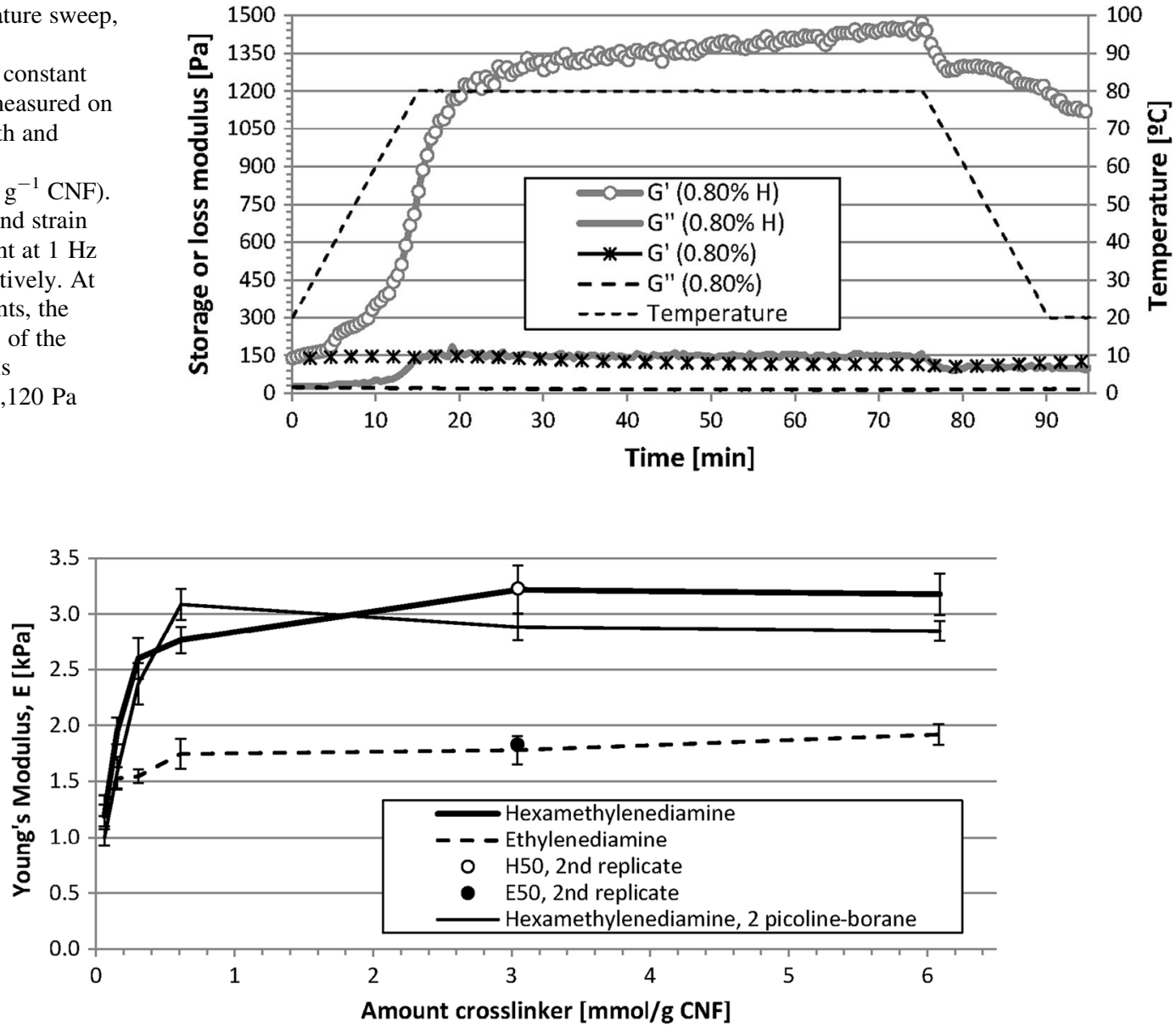

Fig. 3 Young's modulus (E) as a function of crosslinker concentration. Seven gels were prepared and measured for each crosslinker at each concentration. One series was made with crosslinker (HMDA) and 2-picoline-borane. The error bars

of the spacer of the crosslinking molecule plays an important role in the gel strength. Crosslinking with HMDA gives consequently higher values compared to crosslinking with EDA.

The concentration of crosslinker at which the highest $\mathrm{E}$ was obtained (approximately between 0.6 and $3.0 \mathrm{mmol}$ crosslinker per $\mathrm{g} \mathrm{CNF}$ ) is higher than the total amount of aldehyde groups on CNF $\left(0.18 \pm 0.04 \mathrm{mmol} \mathrm{g}^{-1} \mathrm{CNF}\right)$. Each crosslinker molecule carries two amine groups, making the ratio of amine to aldehyde approximately between 7 and 33 . We assume that all aldehyde groups have reacted at these conditions, and that there are crosslinker molecules that have reacted with one amine group only. The resulting gel will thus have an excess of free amine show \pm one standard deviation from the mean. Notice the reproducibility for the second replicates of H50 (3.046 mmol HMDA per g CNF) and E50 (3.042 mmol EDA per g CNF), which were gels prepared independently from the first series

groups. The measured $\mathrm{pH}$ in the gels was around 9, suggesting that both amine groups were protonated and thus positively charged $(\mathrm{pH}<\mathrm{pKa}=11.86$ and 10.76; WolframlAlpha 2014), and that the carboxylic acids were deprotonated and negatively charged $(\mathrm{pH}>\mathrm{pKa}=3-4$; Saito et al. 2011). At such conditions the free amine groups would be available for attractive ionic interaction with carboxyl groups. HMDA have four carbon atoms more in the chain than EDA, and is thus longer and more flexible. The reason why HMDA gives stronger gels compared to EDA is likely to be that more of the HMDA molecules are able to connect to two aldehyde groups (the limiting factor for covalent bonding), i.e. making a functional crosslink between two fibrils. The large increase in gel 

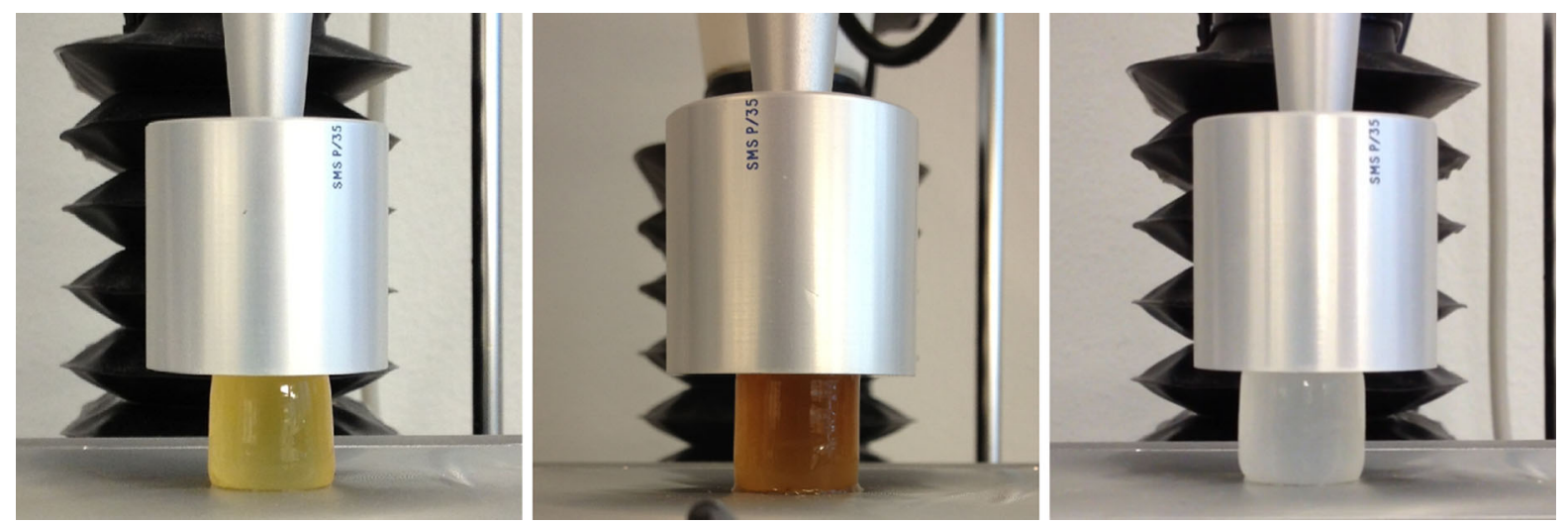

Fig. 4 Gels with different crosslinkers during compression measurements with the texture analyzer. From left to right EDA (E100), HMDA (H50) and HMDA with 2-picoline-borane (H50)

strength obtained by using the longest crosslinker at the same crosslinker concentration is a strong indication of formation of more covalent bonds. The creation of Schiff-bases was also supported by a clear color change in the crosslinked gels from translucent/white to yellow/brownish, which are characteristic colors for Schiff bases (Bartos and Pesez 1979). Using 2-picoline-borane as reductive agent for the Schiff bases changed the color of the gels from yellow/brownish to white, thus indicating the successful reduction. No significant difference in strength due to the reduction of Schiff-bases was observed. The color difference in the gels with HMDA with and without 2-picoline-borane, as well as with EDA can be seen in Fig. 4. The parts of the gel strength originating from ionic interactions versus covalent bonds are not quantified. However, the strong dependence on the crosslinker length indicates that a major part is attributed to the covalent bonds.

The Poisson's ratio $\mu$ gives the relationship between the two elastic moduli ( $\mathrm{E}$ and $\mathrm{G}$ ) as $E=2 G(1+\mu)$, where $-1 \leq \mu \leq 0.5$ for an isotropic material (Tschoegl et al. 2002). When hydrogel materials are fully swollen, their mechanical behavior can be considered similar to rubber-like materials (Anseth et al. 1996), which have a Poisson ratio close to 0.5, and this value is used for e.g. alginate gels (Ahearne et al. 2005). Using this value for the Poisson's ratio and the $\mathrm{G}^{\prime}$ value from the last data points in Fig. 2, approximately $1,120 \mathrm{~Pa}$, the calculated Young's modulus value (E) becomes 3,360 Pa. This corresponds fairly well with the measured E-values for the corresponding $\mathrm{H} 50$ gel of approximately 3,220 $\mathrm{Pa}$.
The Young's moduli of the gels were on the level between brain and muscle tissues (Discher et al. 2005; Reilly and Engler 2010), and can be regarded as soft gels. The relatively low Younǵs modulus is caused by the low solid content of the gels $(0.8 \%)$ and the relative low degree of crosslinking (amount of active sites was $0.17 \mathrm{mmol} \mathrm{CHO}$ per g CNF). As can be seen from Table 2, the gel strength $\left(\mathrm{G}^{\prime}\right)$ of the noncrosslinked CNF dispersion is strongly dependent on the CNF concentration, suggesting that stronger gels can be prepared by increasing the solid content. In addition, the amounts of aldehyde groups can be increased by e.g. periodate oxidation (Chinga-Carrasco and Syverud 2014), providing additional sites for crosslinking and thus stronger gels. The range of

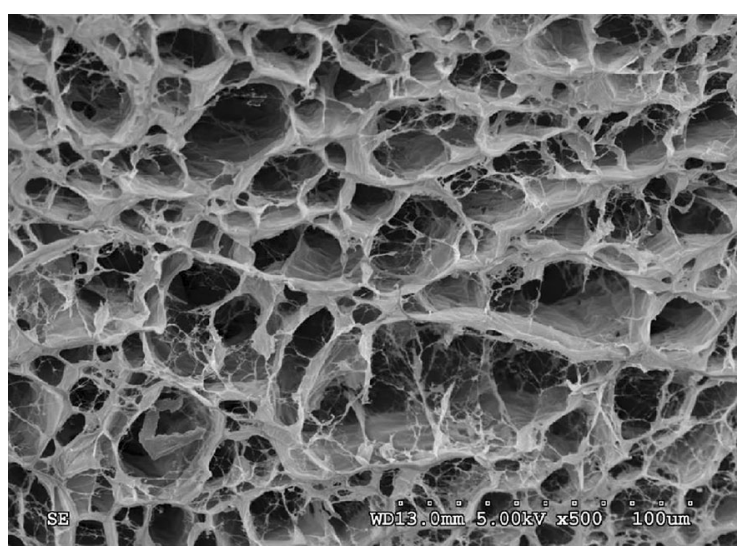

Fig. 5 Freeze dried CNF structure having walls formed by nanofibrils and pores in the micrometer scale 
Fig. 6 Water absorption as a function of time for freeze dried CNF gels without crosslinker and with EDA and HMDA at $3.05 \mathrm{mmol} \mathrm{g}^{-1} \mathrm{CNF}$. The water absorption was estimated according to Eq. 1

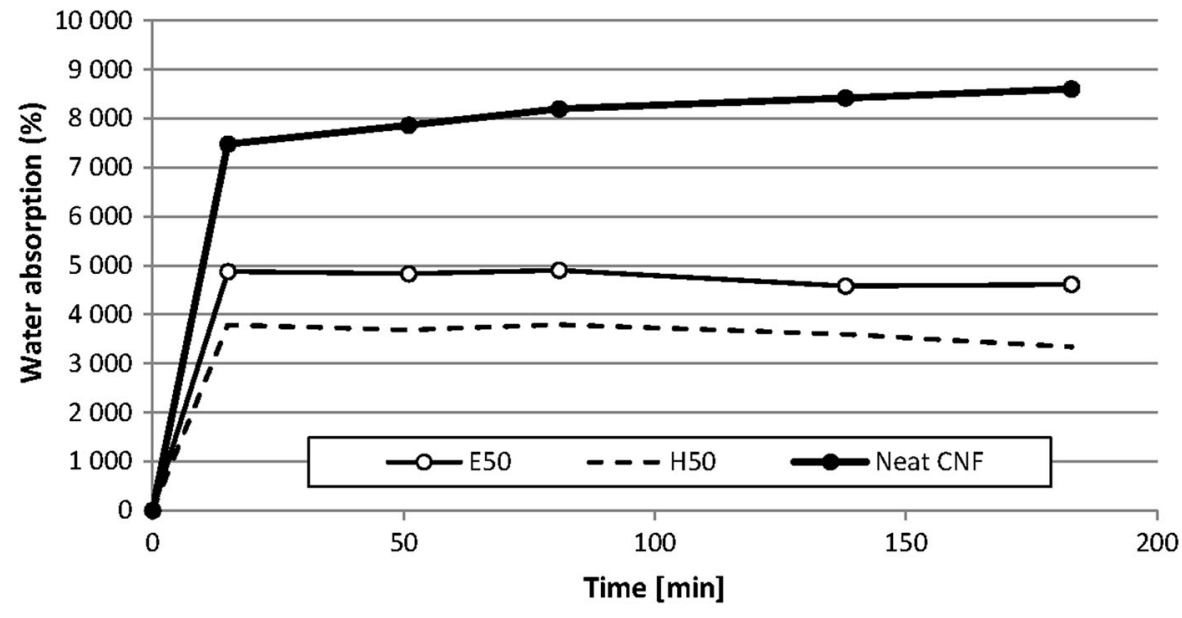

Young's modulus could in principle easily be extended, thus making it possible to design scaffolds fitting the tissue in question.

Some of the gels were freeze dried. During drying, a porous structure with walls of fibrils is formed by creation of numerous hydrogen bonds between neighboring fibrils (Fig. 5). Such pores are in the micrometer range. Keep in mind that the original hydrogel structure with individualized and dispersed CNFs will never be recreated, although the gels are immersed in water. Figure 6 shows the water absorption of the freeze dried gels (E50, H50 and pure CNF) as a function of time. The gels quickly reached stable levels of absorption. The results show that the water absorption is highest for the gel without crosslinker, and lowest for the gel that had the highest Young's modulus, i.e. highest degree of crosslinking. This indicates that by controlling the degree of crosslinking, the degree of water absorption can also be controlled even in these structures, thus preventing an infinite dilution of the gels.

Also important to mention, at the higher crosslinker concentrations, there is a surplus of non-reacted crosslinker molecules. The crosslinked gels are stable when immersed in water, and can even withstand violent magnetic stirring for several minutes without being disintegrated. This suggests that surplus chemicals can be removed by immersing the gels in water and let the chemicals diffuse out of the crosslinked CNF network.

As far as we can consider, both the never dried and the freeze dried structures could be interesting from a tissue engineering point of view. Native ECM has a fibrillar character, and it is expected that the fibrillar structure of the tissue substrate is an advantage (Reilly and Engler 2010). The surfaces forming the walls in the freeze dried structures will also have a certain surface roughness due to the fibrillar structure. The hydroxyl $(\mathrm{OH})$ and carboxyl $(\mathrm{COOH})$ groups on the CNF surfaces will promote; (1) hydrogen bonds between the CNF and cell-surface proteins and (2) ionic interactions between cell membranes and CNF (Seliktar 2012).

\section{Conclusions}

In this work we demonstrate how reversible CNF hydrogels, held together by fibril entanglement, ionic interactions and hydrogen bonds, can be transformed into permanent hydrogels by covalently linking the CNFs. The aldehyde groups formed during TEMPO mediated oxidation were utilized as sites for crosslinking the CNFs using diamines. By varying the concentration and length of the crosslinker molecules, the elastic moduli of the gels were controlled. In tissue engineering, hydrogels are suggested as scaffolds for cells. Their elastic moduli are important for the development of stem cells into differentiated cell types. The possibility to tailor the elastic modulus of CNF hydrogels is thus one important step towards such applications.

Acknowledgments The authors thank Mirjana Filipovic, Ingebjørg Leirset and Anne Marie Reitan (PFI) for skilful laboratory work. The work has been funded by Research Council of Norway through the NANO2021 program, grant no. 219733-NanoHeal: Bio-compatible cellulose nanostructures for advanced wound healing applications, and grant no. 
228147-NORCEL: The Norwegian Nanocellulose Technology Platform.

Open Access This article is distributed under the terms of the Creative Commons Attribution License which permits any use, distribution, and reproduction in any medium, provided the original author(s) and the source are credited.

\section{References}

Ahearne M, Yang Y, El Haj AJ, Then KY, Liu KK (2005) Characterizing the viscoelastic properties of thin hydrogelbased constructs for tissue engineering applications. J R Soc Interface 2:455-463

Alexandrescu L, Syverud K, Gatti A, Chinga-Carrasco G (2013) Cytotoxicity tests of cellulose nanofibril-based structures. Cellulose 20(4):1765-1775

Anseth KS, Bowman CN, Brannon-Peppas L (1996) Review. Mechanical properties of hydrogels and their experimental determination. Biomaterials 17:1647-1657

Barbucci R, Magnani A, Consumi M (2000) Swelling behaviour of carboxymethylcellulose hydrogels in relation to crosslinking, $\mathrm{pH}$ and charge density. Macromolecules 33: 7475-7480

Bartos J, Pesez M (1979) Colorimetric and fluorimetric determination of aldehydes and ketones. Pure Appl Chem 51:1803-1814

Bhattacharya M, Malinen MM, Lauren P, Lou Y-R, Kuisma SW, Kanninen L, Lille M, Corlu A, GuGuen-Guillouzo C, Ikkaka O, Laukkanen A, Urtti A, Yliperttula M (2012) Nanofibrillar cellulose hydrogel promotes three-dimensional liver cell culture. J Control Release 164(3):291-298

Chinga-Carrasco G, Syverud K (2014) Pretreatment-dependent surface chemistry of wood nanocellulose for $\mathrm{pH}$ sensitive hydrogels. J Biomater Appl. doi:10.1177/0885328214531511

Chinga-Carrasco G, Yu Y, Diserud O (2011) Quantitative electron microscopy of cellulose nanofibril structures from Eucalyptus and Pinus radiata pulp fibres. Microsc Microanal 17(4):563-571

Chinga-Carrasco G, Averianova N, Kodalenko O, Garaeva M, Petrov V, Leinsvang B, Karlsen T (2014) The effect of residual fibres on the micro-topography of cellulose nanopaper. Micron 56:80-84

Discher DE, Janmey P, Wang YL (2005) Tissue cells feel and respond to the stiffness of their substrate. Science 310 : $1139-1143$

Engler AJ, Sen S, Sweeney HL, Discher DE (2006) Matrix elasticity directs stem cell lineage specification. Cell 126: 677-689
Fukuzumi H, Saito T, Isogai A (2013) Influence of TEMPOoxidized cellulose nanofibril on film properties. Carbohydr Polym 93:171-177

Han S, Lee M, Kim BK (2010) Crosslinking reactions of oxidized cellulose fiber. I. Reactions between dialdehyde cellulose and multifunctional amines on lyocell fabric. J Appl Polym Sci 117:682-690

Hoffman AS (2001) Hydrogels for biomedical applications. Ann NY Acad Sci 944(1):62-73

Ishii D, Saito T, Isogai A (2011) Viscoelastic evaluation of average length of cellulose nanofibres prepared by TEMPOmediated oxidation. Biomacromolecules 12:548-550

Lou Y-R, Kanninen L, Kuisma T, Niklander J, Noon LA, Burks D, Urtti A, Yliperttula M (2014) The use of nanofibrillar cellulose hydrogel as a flexible three-dimensional model to culture human pluripotent stem cells. Stem Cells Dev 23(4):380-392

Nge TT, Nogi M, Yano H, Sugiyama J (2010) Microstructure and mechanical properties of bacterial cellulose/chitosan porous scaffold. Cellulose 17:349-363

Reilly GC, Engler AJ (2010) Intrinsic extracellular matrix properties regulate stem cell differentiation. J Biomech 43:55-62

Ruhaak LR, Steenvoorden E, Koeleman CAM, Deelder AM, Wuhrer M (2010) 2-Picoline-borane: a non-toxic reducing agent for oligosaccharide labeling by reductive amination. Protemics 10:2330-2336

Saito T, Isogai A (2006) Introduction of aldehyde groups on surfaces of native cellulose fibers by TEMPO-mediated oxidation. Colloid Surf A 289(1-3):219-225

Saito T, Nishiyama Y, Putaux JL, Vignon M, Isogai A (2006) Homogeneous suspensions of individualized microfibrils from TEMPO-catalyzed oxidation of native cellulose. Biomacromolecules 7(6):1687-1691

Saito T, Uematsu T, Kimura S, Enomae T, Isogai A (2011) Selfaligned integration of native cellulose nanofibrils towards producing diverse bulk materials. Soft Matter 7(19):8804-8809

Seliktar D (2012) Designing cell-compatible hydrogels for biomedical applications. Science 336(6085):1124-1128

Syverud K, Kirsebom H, Hajizadeh S, Chinga-Carrasco G (2011) Cross-linking cellulose nanofibrils for potential elastic cryo-structured gels. Nanoscale Res Lett 6:626

Tschoegl NW, Knauss W, Emri I (2002) Poisson's ratio in linear viscoelasticity: a critical review. Mech Time-Depend Mat $6(1): 3-51$

Wolfram|Alpha (2014) WolframlAlpha knowledgebase, Sat Jun 21 17:48:23 CDT 